\title{
Postoperative management of lung transplant recipients
}

\author{
Christina C. Kao, Amit D. Parulekar \\ Section of Pulmonary, Critical Care, and Sleep Medicine, Department of Medicine, Baylor College of Medicine, Houston, TX, USA \\ Contributions: (I) Conception and design: All authors; (II) Administrative support: None; (III) Provision of study materials or patients: None; (IV) \\ Collection and assembly of data: None; (V) Data analysis and interpretation: None; (VI) Manuscript writing: All authors; (VII) Final approval of \\ manuscript: All authors. \\ Correspondence to: Amit D. Parulekar, MD, MS. Section of Pulmonary, Critical Care, and Sleep Medicine, Department of Medicine, Baylor College of \\ Medicine, 6620 Main Street, Suite 11C.14, Houston, TX 77030, USA. Email: paruleka@bcm.edu.
}

\begin{abstract}
Despite advances in surgical technique, lung transplantation is associated with worse survival when compared with other solid organ transplantations. Graft dysfunction and infection are the leading causes of mortality in the first 30 days following transplantation. Primary graft dysfunction (PGD) is a form of reperfusion injury that occurs early after transplantation. Management of PGD is mainly supportive with use of lung protective ventilation. Inhaled nitric oxide (iNO) and extracorporeal membrane oxygenation may be used in severe cases. Bacterial pneumonias are the most common infectious complication in the immediate post transplant period, but invasive fungal infections may also occur. Other potential complications in the postoperative period include atrial arrhythmias and neurologic complications such as stroke. There is a lack of multicenter, randomized trials to guide ventilation strategies, infection prophylaxis, and treatment of atrial arrhythmias, therefore prevention and management of post-transplant complications vary by transplant center.
\end{abstract}

Keywords: Primary graft dysfunction (PGD); ventilation; arrhythmia

Submitted Apr 02, 2019. Accepted for publication May 21, 2019.

doi: $10.21037 /$ jtd.2019.05.60

View this article at: http://dx.doi.org/10.21037/jtd.2019.05.60

\section{Introduction}

Survival after lung transplantation is inferior to that of other solid organ transplants. Although short-term (30-day and 1-year) survival have improved steadily over recent eras, 5 -year survival has not changed significantly in the two eras from 1999-2008 and 2009-2015 (1). Improvement in shortterm survival is likely a result of both improved surgical techniques and better management in the immediate postoperative period. The most common causes of death in the first 30 days after transplant are graft failure, accounting for $24.3 \%$ of deaths, and infection, accounting for $19.2 \%$ of deaths (1). Chronic lung allograft dysfunction (CLAD) remains the leading cause of death after the first year post lung transplantation. This article will review the early postoperative complications following lung transplantation including primary graft dysfunction (PGD), arrhythmias, infection, and non-pulmonary organ failure.

\section{Early management of the lung allograft}

\section{$P G D$}

PGD is a form of lung injury that occurs early after lung transplant and is characterized by diffuse alveolar infiltrates on chest radiograph and hypoxemia. In 2005, the International Society for Heart and Lung Transplantation (ISHLT) standardized the definition of PGD and developed a classification system. This classification was recently updated in 2016 (2). PGD severity is graded from 0 to 3 based on the presence of radiographic lung infiltrates and the ratio of alveolar oxygenation $\left(\mathrm{PaO}_{2}\right)$ to fraction of inspired oxygen (FiO2) as shown in Table 1. The 2016 update allows oxygen saturation $\left(\mathrm{SaO}_{2}\right)$ to be substituted if $\mathrm{PaO}_{2}$ is not available. The need for extracorporeal membrane oxygenation (ECMO) with pulmonary edema on $\mathrm{X}$-ray is considered grade 3. For patients on ECMO 
Table 1 Classification of primary graft dysfunction

\begin{tabular}{lccc}
\hline Grade & $\begin{array}{c}\text { Pulmonary edema } \\
\text { on chest X-ray }\end{array}$ & $\mathrm{PaO}_{2} / \mathrm{FiO}_{2}$ ratio & $\mathrm{SaO}_{2} / \mathrm{FiO}_{2}$ ratio \\
\hline 0 & No & Any & Any \\
1 & Yes & $>300$ & $>315$ \\
2 & Yes & $200-300$ & $235-315$ \\
3 & Yes & $<200$ & $<235$ \\
\hline
\end{tabular}

$\mathrm{PaO}_{2}$, partial pressure of oxygen; $\mathrm{FiO}_{2}$, fraction of inspired oxygen; $\mathrm{SaO}_{2}$, oxygen saturation.

without pulmonary edema, PGD is ungradable. Patients on mechanical ventilation and requiring nitric oxide are graded based on the standard classification. PGD timing starts at the point of the reperfusion of the second lung (T0), and grading is recommended at 24,48 , and 72 hours.

The incidence of PGD grade 3 at any point during the first 72 hours is reported to be approximately 30 percent. The development of PGD is associated with poor shortterm and long-term outcomes. PGD is associated with increased 30- and 90-day mortality as well as worse survival after 1 year $(3,4)$. It is also a major risk factor for the development of bronchiolitis obliterans syndrome (BOS), the most common form of CLAD (4-6). Although PGD grades 1 and 2 have been associated with increased mortality and development of BOS, PGD grade 3 is more strongly associated with worse outcomes $(5,6)$.

A number of risk factors for the development of PGD have been identified, including donor and recipient characteristics as well as intra-operative factors. Modification of risk factors, when possible, may help to prevent PGD. For example, allografts from donors with current or former smoking exposure increase PGD risk (7). However, while use of donors with smoking exposure leads to worse outcomes, complete avoidance of such donor lungs would increase waitlist mortality (8). Therefore, limiting use of lungs from donors with smoking exposure to lower risk recipients may help to decrease the risk for PGD. Undersized lung allografts have also been shown to be associated with a higher risk of PGD (9). Therefore, attention to size matching may help to minimize risk of PGD. Body mass index (BMI) is an important modifiable recipient risk factor. Overweight and obesity have both been associated with risk of PGD $(7,10)$. Thus, weight loss should be stressed in potential lung transplant candidates who are overweight.

Treatment for PGD is mainly supportive, including use of lung-protective strategy with low tidal volume ventilation and a restrictive fluid strategy based on evidence extrapolated from management of acute respiratory distress syndrome. Currey et al. demonstrated that grade of PGD was lower after introduction of a respiratory and hemodynamic management guideline which targeted a central venous pressure less than or equal to 7 and a tidal volume of $6 \mathrm{~mL} / \mathrm{kg}$ in patients with hypoxemia (11). Although inhaled nitric oxide (iNO) is not effective for preventing the development of PGD (12), it may help improve oxygenation in patients with severe hypoxemia $(13,14)$. ECMO has also been used in patients with severe PGD when gas exchange is inadequate with mechanical ventilation alone. In addition, utilization of early ECMO to limit sedation, neuromuscular blockade, and ventilatorinduced lung injury has the potential to improve outcomes in patients with severe PGD. Analysis of data from the Extracorporeal Life Support Organization (ELSO) registry found that 151 post lung transplant patients were on either venovenous or venoarterial ECMO for PGD, and hospital survival was only $42 \%$ (15). In a more recent single center report of patients who required ECMO for PGD, survival was $82 \%$ at 30 days and $64 \%$ at one year, but maximum $\mathrm{FEV}_{1}$ post transplant was significantly worse in patients who required ECMO compared with those who did not (16).

\section{Mechanical ventilation}

There is increasing evidence for the beneficial effects of low tidal volume ventilation in patients without acute respiratory distress syndrome (17). There are no large, multicenter trials to guide ventilator management following lung transplantation, and an international survey aimed to assess mechanical ventilation practices after lung transplant demonstrated wide variation in practices (18). Although low tidal ventilation $(6 \mathrm{~mL} / \mathrm{kg}$ of predicted body weight) was the preferred strategy, tidal volume was most commonly determined by recipient characteristics. In fact, donor characteristics were frequently not even known by the team managing the ventilator. Oversized allografts are associated with a decreased risk of PGD grade 3 (19), which may be because oversized allografts are ventilated with smaller tidal volumes in $\mathrm{mL} / \mathrm{kg}$-donor-predicted body weights compared with undersized allografts (20). The optimal method to increase oxygenation post lung transplantation is also unclear. High $\mathrm{FiO}_{2}$ has potential toxicity, but the maximum acceptable PEEP is also unclear. It is therefore unknown whether minimizing $\mathrm{FiO}_{2}$ versus PEEP is the better 
strategy. In patients undergoing single lung transplant, the native lung disease can also affect decisions regarding mechanical ventilation. Ventilation strategies for interstitial lung disease differ than those for emphysema given the risk of native lung hyperinflation in single lung transplants for emphysema.

\section{Fluid management}

Postoperatively, transplanted lungs have varying degrees of pulmonary edema as a result of increased vascular permeability and severed lymphatic drainage (21). Therefore, a fluid restrictive management strategy may be useful in limiting pulmonary edema. At the same time, fluid resuscitation may be necessary to maintain an adequate blood pressure and optimized organ perfusion. Common causes of hypotension in the postoperative period include systemic inflammatory response syndrome as a result of the surgery or use of cardiopulmonary bypass, medicationinduced hypotension, hemorrhage, tamponade, and heart failure (22).

\section{Infectious complications}

Timing is a major factor in the development of infections after lung transplantation. In the first 30 days after transplant, infections are the second leading cause of death (accounting for $19.2 \%$ of deaths in the first 30 days), whereas from 30 days to 1 year after transplant, infections are the leading cause of death (1). Types of infections are also time dependent with nosocomial infections being more common early after transplant.

Bacterial pneumonia is the most frequent infectious complication following lung transplantation (23). Early episodes of pneumonia are often caused by microorganisms cultured from donor lungs (24), but nosocomial infections are also common. The highest risk of pneumonia occurs in the first 30 days after transplantation and risk decreases after 6 months (25) Bloodstream infections are also common, affecting approximately $10 \%$ of patients in one series (26) and was associated with $25 \%$ mortality at 28 days in another series (27). Pseudomonas aeruginosa and Staphylococcus aureus were the most common organisms isolated (27). Cultures from blood, urine, and sputum should be obtained early in an infectious workup. Antibiotic coverage should initially be broad, including coverage for organisms isolated from donor lungs, then tapered based on isolated pathogens and results of susceptibility testing. Patients with chronic infections, such as patients with cystic fibrosis, should receive antibiotics based on pre-transplant cultures and sensitivities.

Viral infections are uncommon in the immediate postoperative period. Cytomegalovirus (CMV) infection is a frequently encountered viral infection in lung transplant patients; however, mortality directly related to CMV disease is rare in the modern era (1). The risk of CMV infection is influenced by the immune status of the donor and recipient and the use of lymphocyte-depletion therapy. Antiviral prophylaxis, generally with IV ganciclovir followed by oral valganciclovir, reduces CMV disease and infection (28), and CMV prophylaxis is generally started in the immediate postoperative period.

Fungal infections are common in lung transplant recipients and are associated with significant morbidity and mortality. The majority of invasive fungal infections occur more than 90 days after transplantation, particularly within the first 3 to 12 months (29). The incidence of invasive fungal infection in lung transplant patients was found to be $8.6 \%$ in the first year with invasive Aspergillus being the most frequent fungal pathogen and Candida being the second most common (30). Candida species are responsible for most infections that occur in the first month post transplant, while the majority of fungal infections from 1 to 6 months post transplant are due to Aspergillus species (31). There is no widely accepted optimal method for prevention of fungal infections. However, the majority of transplant centers use antifungal prophylaxis, though prophylactic strategy includes both a universal approach and a targeted approach to selected patients $(32,33)$. Antifungal agents used for prophylaxis include itraconazole, fluconazole, inhaled amphotericin B, voriconazole, micafungin, and caspofungin (31).

\section{Atrial arrhythmias}

Atrial arrhythmias including atrial fibrillation, atrial tachycardia, and atrial flutter are common after lung transplantation. They occur in $19-46 \%$ of patients in the early postoperative period (34-39) with a peak onset between 2 and 7 days after transplant (40-42). Risk factors for early postoperative atrial tachyarryhthmias include age, history of pretransplant atrial tachyarrhythmia, male gender, hypertension, hyperlipidemia, coronary artery disease, left atrial enlargement, pulmonary fibrosis, and postoperative vasopressor use $(34,35,39-43)$. The impact of pulmonary artery pressures and type of transplant (single versus double) remain controversial (36). 
Potential mechanisms of early postoperative atrial tachyarrhythmia include operative trauma with systemic inflammation and pericarditis, fluid shifts, electrolyte abnormalities, autonomic triggers, and hemodynamic changes (36). Additionally, arrhythmogenesis from the remnant recipient left atrial and pulmonic vein tissue may be a major source of atrial arrhythmia following lung transplantation (34).

Atrial tachyarrhythmias may have a significant impact on post-transplant outcomes. Studies have shown an average increase in post-transplant length of stay of 8-10 days in patients with atrial arrhythmias $(37,39)$. Individual studies have reached mixed conclusions regarding the impact of atrial arrhythmias on mortality after lung transplantation. However, two recent meta-analyses have shown an increase in mortality in patients with atrial arrhythmia following lung transplantation. Waldon et al. showed a higher odds of perioperative mortality (OR: 2.70; 95\% CI: 1.73-4.19, $\mathrm{P}<0.001$ ) (37). Similarly, Fan et al. showed an association between postoperative atrial arrhythmia and lower overall survival (OR: 1.72; 95\% CI: 1.39-2.12, $\mathrm{P}<0.001)$ (39). It is important to note that atrial arrhythmias may not play a causative role in increased mortality. They may in fact be markers of contributions of increased age, comorbidities, and perioperative complications.

There are no well-established guidelines for prevention and management of atrial arrhythmias following lung transplantation. Attention should be given to fluid management with a focus on avoidance of volume overload. Early postoperative initiation of beta blockers in hemodynamically stable patients, especially those with preoperative atrial arrhythmia or beta blocker use, should be considered. When atrial arrhythmias occur, our center favors an initial trial of rate control with beta blocker or calcium channel blocker. If patients are hemodynamically unstable, cardioversion is performed. Rhythm control with cardioversion or medications such as amiodarone is considered when rate control is ineffective. In patients with persistent or recurrent atrial arrhythmias, we initiate anticoagulation within 24-48 hours as long as there are no concerns for surgical bleeding. Optimal duration of antiarrhythmic drugs and anticoagulation is not welldefined. We favor limiting duration of antiarrhythmic therapy to 4-6 weeks in patients without pretransplant atrial arrhythmia. We also limit use of anticoagulation in patients with new onset atrial arrhythmia, especially if the arrhythmia is transient and normal sinus rhythm is maintained. In patients with persistent or recurrent arrhythmias or pretransplant arrhythmia, longer-term anticoagulation may be necessary.

\section{Neurologic complications}

Neurologic complications are common early after lung transplantation. In a single center retrospective review of 700 lung transplant recipients, $9.2 \%$ of patients developed major neurologic complications within 2 weeks after transplantation (44). Stroke (41\%) and severe metabolic encephalopathy were the most common complications. Patients with neurologic complications had higher 90-day mortality when compared with patients without neurologic complications $(15 \%$ versus $4 \%, \mathrm{P}=0.03)$. Multivariate analysis showed age, coronary artery disease, prolonged cardiopulmonary bypass, and PGD were risk factors for death associated with neurologic complications, suggesting neurologic complications may be a surrogate for a worse postoperative course. These findings highlight the importance of close neurologic monitoring as an important component of post transplantation management. Attention should also focus on minimization of excess sedation and management of medication side effects, drug interactions, and sleep cycles.

Severe hyperammonemia occurs in the first 2 weeks after transplant in approximately $1 \%$ of lung transplant recipients (45). Symptoms may include somnolence, lethargy, confusion, or agitation. Mortality has been reported to be as high as $75 \%$ in small case series (45). Early clinical recognition of symptoms and confirmation of diagnosis with plasma ammonia levels are imperative for early intervention. Treatment consists of early initiation of aggressive hemodialysis with high intensity and frequency. Less efficient modes of dialysis are ineffective at lowering ammonia levels. Other treatments include reduction of immunosuppression, protein restriction, bowel decontamination, and other agents aimed at activating alternative nitrogen pathways. More recently, reports have suggested a link between urea-splitting organisms and hyperammonemia $(46,47)$. For this reason, we favor empiric addition of a fluoroquinolone and macrolide to cover such organisms. In our experience, the above interventions have resulted in lower mortality compared with reported cases.

\section{Conclusions}

Despite improvements in perioperative management of lung transplant recipients, significant complications may arise 
in the early post transplant period. PGD is common and requires high-quality supportive care, appropriate fluid and ventilator management, and early consideration of ECMO support when appropriate. Infectious complications, atrial arrhythmias, and neurologic complications are also common and require vigilance by the care team to ensure early detection and intervention. Given the lack of clinical trial data and multicenter center studies, early post transplant management varies amongst transplant centers and practitioners.

\section{Acknowledgments}

None.

\section{Footnote}

Conflicts of Interest: The authors have no conflicts of interest to declare.

\section{References}

1. Chambers DC, Yusen RD, Cherikh WS, et al. The Registry of the International Society for Heart and Lung Transplantation: Thirty-fourth Adult Lung And HeartLung Transplantation Report-2017; Focus Theme: Allograft ischemic time. J Heart Lung Transplant 2017;36:1047-59.

2. Snell GI, Yusen RD, Weill D, et al. Report of the ISHLT Working Group on Primary Lung Graft Dysfunction, part I: Definition and grading-A 2016 Consensus Group statement of the International Society for Heart and Lung Transplantation. J Heart Lung Transplant 2017;36:1097-103.

3. Christie JD, Kotloff RM, Ahya VN, et al. The effect of primary graft dysfunction on survival after lung transplantation. Am J Respir Crit Care Med 2005;171:1312-6.

4. Kreisel D, Krupnick AS, Puri V, et al. Short- and longterm outcomes of 1000 adult lung transplant recipients at a single center. J Thorac Cardiovasc Surg 2011;141:215-22.

5. Daud SA, Yusen RD, Meyers BF, et al. Impact of immediate primary lung allograft dysfunction on bronchiolitis obliterans syndrome. Am J Respir Crit Care Med 2007;175:507-13.

6. Whitson BA, Prekker ME, Herrington CS, et al. Primary graft dysfunction and long-term pulmonary function after lung transplantation. J Heart Lung Transplant
2007;26:1004-11.

7. Diamond JM, Lee JC, Kawut SM, et al. Clinical risk factors for primary graft dysfunction after lung transplantation. Am J Respir Crit Care Med 2013;187:527-34.

8. Bonser RS, Taylor R, Collett D, et al. Effect of donor smoking on survival after lung transplantation: a cohort study of a prospective registry. Lancet 2012;380:747-55.

9. Eberlein M, Arnaoutakis GJ, Yarmus L, et al. The effect of lung size mismatch on complications and resource utilization after bilateral lung transplantation. J Heart Lung Transplant 2012;31:492-500.

10. Lederer DJ, Kawut SM, Wickersham N, et al. Obesity and primary graft dysfunction after lung transplantation: the Lung Transplant Outcomes Group Obesity Study. Am J Respir Crit Care Med 2011;184:1055-61.

11. Currey J, Pilcher DV, Davies A, et al. Implementation of a management guideline aimed at minimizing the severity of primary graft dysfunction after lung transplant. J Thorac Cardiovasc Surg 2010;139:154-61.

12. Botha P, Jeyakanthan $M$, Rao JN, et al. Inhaled nitric oxide for modulation of ischemia-reperfusion injury in lung transplantation. J Heart Lung Transplant 2007;26:1199-205.

13. Adatia I, Lillehei C, Arnold JH, et al. Inhaled nitric oxide in the treatment of postoperative graft dysfunction after lung transplantation. Ann Thorac Surg 1994;57:1311-8.

14. Date H, Triantafillou AN, Trulock EP, et al. Inhaled nitric oxide reduces human lung allograft dysfunction. J Thorac Cardiovasc Surg 1996;111:913-9.

15. Fischer S, Bohn D, Rycus P, et al. Extracorporeal membrane oxygenation for primary graft dysfunction after lung transplantation: analysis of the Extracorporeal Life Support Organization (ELSO) registry. J Heart Lung Transplant 2007;26:472-7.

16. Hartwig MG, Walczak R, Lin SS, et al. Improved survival but marginal allograft function in patients treated with extracorporeal membrane oxygenation after lung transplantation. Ann Thorac Surg 2012;93:366-71.

17. Neto AS, Simonis FD, Barbas CS, et al. Lung-Protective Ventilation With Low Tidal Volumes and the Occurrence of Pulmonary Complications in Patients Without Acute Respiratory Distress Syndrome: A Systematic Review and Individual Patient Data Analysis. Crit Care Med 2015;43:2155-63.

18. Beer A, Reed RM, Bolukbas S, et al. Mechanical ventilation after lung transplantation. An international survey of practices and preferences. Ann Am Thorac Soc 
2014;11:546-53.

19. Eberlein M, Reed RM, Bolukbas S, et al. Lung size mismatch and primary graft dysfunction after bilateral lung transplantation. J Heart Lung Transplant 2015;34:233-40.

20. Dezube R, Arnaoutakis GJ, Reed RM, et al. The effect of lung-size mismatch on mechanical ventilation tidal volumes after bilateral lung transplantation. Interact Cardiovasc Thorac Surg 2013;16:275-81.

21. Siegleman SS, Sinha SB, Veith FJ. Pulmonary reimplantation response. Ann Surg 1973;177:30-6.

22. Fuehner T, Kuehn C, Welte T, et al. ICU Care Before and After Lung Transplantation. Chest 2016;150:442-50.

23. Nakajima T, Palchevsky V, Perkins DL, et al. Lung transplantation: infection, inflammation, and the microbiome. Semin Immunopathol 2011;33:135-56.

24. Speich R, van der Bij W. Epidemiology and management of infections after lung transplantation. Clin Infect Dis 2001;33 Suppl 1:S58-65.

25. Aguilar-Guisado M, Givalda J, Ussetti P, et al. Pneumonia after lung transplantation in the RESITRA Cohort: a multicenter prospective study. Am J Transplant 2007;7:1989-96.

26. Valentine VG, Bonvillain RW, Gupta MR, et al. Infections in lung allograft recipients: ganciclovir era. J Heart Lung Transplant 2008;27:528-35.

27. Husain S, Chan KM, Palmer SM, et al. Bacteremia in lung transplant recipients in the current era. Am J Transplant 2006;6:3000-7.

28. Zamora MR, Nicolls MR, Hodges TN, et al. Following universal prophylaxis with intravenous ganciclovir and cytomegalovirus immune globulin, valganciclovir is safe and effective for prevention of CMV infection following lung transplantation. Am J Transplant 2004;4:1635-42.

29. Kennedy CC, Razonable RR. Fungal Infections After Lung Transplantation. Clin Chest Med 2017;38:511-20.

30. Pappas PG, Alexander BD, Andes DR, et al. Invasive fungal infections among organ transplant recipients: results of the Transplant-Associated Infection Surveillance Network (TRANSNET). Clin Infect Dis 2010;50:1101-11.

31. Neoh CF, Snell G, Levvey B, et al. Antifungal prophylaxis in lung transplantation. Int J Antimicrob Agents 2014;44:194-202.

32. He SY, Makhzoumi ZH, Singer JP, et al. Practice variation in Aspergillus prophylaxis and treatment among lung transplant centers: a national survey. Transpl Infect Dis 2015;17:14-20.
33. Neoh CF, Snell GI, Kotsimbos T, et al. Antifungal prophylaxis in lung transplantation--a world-wide survey. Am J Transplant 2011;11:361-6.

34. Chaikriangkrai K, Jyothula S, Jhun HY, et al. Incidence, Risk Factors, Prognosis, and Electrophysiological Mechanisms of Atrial Arrhythmias after Lung Transplantation. JACC Clin Electrophysiol 2015;1:296-305.

35. Nielsen TD, Bahnson T, Davis RD, et al. Atrial fibrillation after pulmonary transplant. Chest 2004;126:496-500.

36. Roukoz H, Benditt DG. Atrial arrhythmias after lung transplantation. Trends Cardiovasc Med 2018;28:53-61.

37. Waldron NH, Klinger RY, Hartwig MG, et al. Adverse outcomes associated with postoperative atrial arrhythmias after lung transplantation: A meta-analysis and systematic review of the literature. Clin Transplant 2017;31.

38. Dizon JM, Chen K, Bacchetta M, et al. A comparison of atrial arrhythmias after heart or double-lung transplantation at a single center: insights into the mechanism of post-operative atrial fibrillation. J Am Coll Cardiol 2009;54:2043-8.

39. Fan J, Zhou K, Li S, et al. Incidence, risk factors and prognosis of postoperative atrial arrhythmias after lung transplantation: a systematic review and meta-analysis. Interact Cardiovasc Thorac Surg 2016;23:790-9.

40. Henri C, Giraldeau G, Dorais M, et al. Atrial fibrillation after pulmonary transplantation: incidence, impact on mortality, treatment effectiveness, and risk factors. Circ Arrhythm Electrophysiol 2012;5:61-7.

41. Mason DP, Marsh DH, Alster JM, et al. Atrial fibrillation after lung transplantation: timing, risk factors, and treatment. Ann Thorac Surg 2007;84:1878-84.

42. Orrego CM, Cordero-Reyes AM, Estep JD, et al. Atrial arrhythmias after lung transplant: underlying mechanisms, risk factors, and prognosis. J Heart Lung Transplant 2014;33:734-40.

43. D'Angelo AM, Chan EG, Hayanga JW, et al. Atrial arrhythmias after lung transplantation: Incidence and risk factors in 652 lung transplant recipients. J Thorac Cardiovasc Surg 2016;152:901-9.

44. Shigemura N, Sclabassi RJ, Bhama JK, et al. Early major neurologic complications after lung transplantation: incidence, risk factors, and outcome. Transplantation 2013;95:866-71.

45. Chen C, Bain KB, Iuppa JA, et al. Hyperammonemia Syndrome After Lung Transplantation: A Single Center Experience. Transplantation 2016;100:678-84.

46. Bharat A, Cunningham SA, Scott Budinger GR, et 
al. Disseminated Ureaplasma infection as a cause of fatal hyperammonemia in humans. Sci Transl Med 2015;7:284re3.

Cite this article as: Kao CC, Parulekar AD. Postoperative management of lung transplant recipients. J Thorac Dis 2019;11(Suppl 14):S1782-S1788. doi: 10.21037/jtd.2019.05.60
47. Wylam ME, Kennedy CC, Hernandez NM, et al. Fatal hyperammonaemia caused by Mycoplasma hominis. Lancet 2013;382:1956. 